\title{
MAP9 single nucleotide polymorphism rs1058992 is a risk of EBV-associated gastric carcinoma in Chinese population
}

\author{
W. $\mathrm{LIU}^{1}, \mathrm{H} . \mathrm{XIAO}^{1}, \mathrm{~S} . \mathrm{WU}{ }^{1}, \mathrm{H} . \mathrm{LIU}^{2}, \mathrm{~B} . \mathrm{LUO}^{1 *}$
}

\begin{abstract}
${ }^{1}$ Department of Pathogenic Biology, Faculty of Medicine, Qingdao University, 38 Dengzhou Road, Qingdao, 266021, P. R. China; ${ }^{2}$ Department of Clinical Laboratory, Central Hospital of Zibo, 54 Gongqingtuan Road, ZiBo, 255036, P. R. China
\end{abstract}

\begin{abstract}
Summary. - Microtubule-associated protein 9 (MAP9) is a mitosis-associated protein involved in bipolar spindle assembly. Following DNA damage, MAP9 stabilizes p53 via p300 and MDM2 (mouse double minute-2 homolog). The dysregulation of MAP9 was considered to be associated with tumorigenesis. Single nucleotide polymorphisms (SNPs) in key genes governing mitosis may particularly increase susceptibility to gastric carcinoma (GC). Our study demonstrated that the CC homozygous genotype of SNP rs1058992 located in the MAP9 gene was significantly correlated with EBV-associated GC (EBVaGC) in a recessive genetic model $(\mathrm{OR}=2.558,95 \% \mathrm{CI}=1.306-5.010, P=0.043)$, and the $\mathrm{C}$ allele frequency of rs 1058992 also showed significant correlation with EBVaGC $(\mathrm{OR}=1.904,95 \% \mathrm{CI}=1.141-3.179, P=0.013)$. These results suggest that the MAP9 rs 1058992 polymorphism is associated with risk of EBVaGC. The conversion of lysine to arginine caused by rs1058992 may affect development of EBVaGC; however, further studies in larger populations are needed to fully elucidate its role in EBVaGC.
\end{abstract}

Keywords: SNP; EBV; gastric carcinoma; MAP9

\section{Introduction}

Epstein-Barr virus (EBV) is a well-known oncogenic virus that infects more than $95 \%$ of the global population (Ali et al., 2015). Numerous studies have shown that EBV is closely related to tumor occurrence (Ko, 2015). EBV infection is particularly associated with the development of a variety of malignancies of epithelial and lymphoid origin, including gastric carcinoma (GC), nasopharyngeal carcinoma (NPC), Burkitt's lymphoma, and Hodgkin's disease (Huang et al., 2014; Rosales-Perez et al., 2014; Tanyildiz et al., 2015; Wu et al., 2016). The ability of EBV to immortalize B cells demon-

*Corresponding author. E-mail: qdluobing@163.com; phone: +86532-82991083.

Abbreviations: AURKA = aurora kinase A; EBER1 = EBV-encoded small RNA 1; EBV = Epstein-Barr virus; $\mathrm{EBVaGC}=\mathrm{EBV}$ associated gastric carcinoma; $\mathrm{EBVnGC}=\mathrm{EBV}$ negative gastric carcinoma; $\mathrm{GC}=$ gastric carcinoma; MAP9 $=$ microtubule associated protein 9; NPC = nasopharyngeal carcinoma; Plk1 = polo-like kinase 1 ; $\mathrm{SNP}=$ single nucleotide polymorphism strates its important role in the pathogenesis of lymphoma (Young and Rickinson, 2004). More notably, EBV infection is detected in nearly $100 \%$ of non-keratinizing nasopharyngeal carcinomas (NPCs) (Elgui de Oliveira et al., 2016). However, the oncogenesis of EBV-associated malignancies is not entirely understood.

Gastric cancer is the fifth leading cause of cancer and the second leading cause of cancer-related deaths worldwide (Camargo et al., 2014; Ferlay et al., 2015). The tumorigenesis of gastric cancer is a multistep process, involving numerous factors. EBV latent infection and persistence in cells is believed to be one of these factors. On the basis of its molecular characteristics, EBV-associated gastric cancer (EBVaGC) has been classified as a distinct molecular subtype of GC, separate from GC with microsatellite instability, genomically stable GC, and GC with chromosomal instability (Cancer Genome Atlas Research, 2014). Approximately 10\% of GCs are EBV positive (Elgui de Oliveira et al., 2016). Because of the incidence of GC, this is a significant number of patients; thus, the pathogenesis of EBVaGC warrants further investigation. 
Table 1. Genotype and allele frequencies of the MAP9 polymorphism for gastric cancer patients and normal controls

\begin{tabular}{|c|c|c|c|}
\hline MAP9/rs1058992 & $\begin{array}{c}\text { EBVnGC }(\mathbf{n}=94) \\
n(\%)\end{array}$ & $\begin{array}{c}\mathrm{NC}(\mathrm{n}=100) \\
\mathrm{n}(\%)\end{array}$ & $\begin{array}{c}\text { EBVaGC }(\mathbf{n}=58) \\
n(\%)\end{array}$ \\
\hline \multicolumn{4}{|l|}{ genotype } \\
\hline TT & $11(11.70)$ & $14(14.00)$ & $4(6.90)$ \\
\hline CT & $51(54.26)$ & $45(45.00)$ & $21(36.20)$ \\
\hline OR $(95 \% \mathrm{CI})$ & $1.442(0.595-3.497)^{\mathrm{a}}$ & $1.132(0.324-3.961)^{c}$ & $1.633(0.479-5.566)^{\mathrm{b}}$ \\
\hline $\mathrm{P}$ & $0.416^{\mathrm{a}}$ & $1.000^{c}$ & $0.430^{\mathrm{b}}$ \\
\hline $\mathrm{CC}$ & $32(34.04)$ & $41(41.00)$ & $33(56.90)$ \\
\hline OR $(95 \% \mathrm{CI})$ & $0.993(0.398-2.480)^{\mathrm{a}}$ & $2.836(0.818-9.833)^{c}$ & $2.817(0.847-9.372)^{b}$ \\
\hline$P$ & $0.989^{\mathrm{a}}$ & $0.091^{\mathrm{c}}$ & $0.083^{\mathrm{b}}$ \\
\hline \multicolumn{4}{|l|}{ recessive model } \\
\hline $\mathrm{TT}+\mathrm{CT}$ & $62(65.96)$ & $59(59.00)$ & $25(43.10)$ \\
\hline CC & $32(34.04)$ & $41(41.00)$ & $33(56.90)$ \\
\hline OR $(95 \% \mathrm{CI})$ & $0.743(0.414-1.332)^{\mathrm{a}}$ & $2.558(1.306-5.010)^{c}$ & $1.900(0.987-3.656)^{b}$ \\
\hline$P$ & $0.317^{\mathrm{a}}$ & $0.006^{c *}$ & $0.054^{\mathrm{b}}$ \\
\hline \multicolumn{4}{|l|}{ dominant model } \\
\hline TT & $11(11.70)$ & $14(14.00)$ & $4(6.90)$ \\
\hline $\mathrm{CC}+\mathrm{CT}$ & $83(88.30)$ & $86(86.00)$ & $54(93.10)$ \\
\hline OR $(95 \% \mathrm{CI})$ & $0.711(0.294-1.721)^{\mathrm{a}}$ & $1.789(0.542-5.908)^{c}$ & $2.779(0.865-8.930)^{b}$ \\
\hline$P$ & $0.449^{\mathrm{a}}$ & $0.335^{\mathrm{c}}$ & $0.076^{\mathrm{b}}$ \\
\hline \multicolumn{4}{|l|}{ allele } \\
\hline $\mathrm{T}$ & $73(38.83)$ & $73(36.50)$ & $29(25.00)$ \\
\hline $\mathrm{C}$ & $115(61.17)$ & $127(63.50)$ & $87(75.00)$ \\
\hline OR $(95 \% \mathrm{CI})$ & $0.906(0.600-1.366)^{\mathrm{a}}$ & $1.904(1.141-3.179)^{c}$ & $1.724(1.036-2.870)^{b}$ \\
\hline$P$ & $0.636^{\mathrm{a}}$ & $0.013^{\text {c* }}$ & $0.035^{b *}$ \\
\hline
\end{tabular}

EBVaGC: EBV-associated gastric carcinoma; EBVnGC: EBV-negative gastric carcinoma; NC: normal control; OR: odds ratio; $95 \%$ CI: $95 \%$ confidence interval; a: EBVnGC vs NC; b: EBVaGC vs NC; c: EBVaGC vs. EBVnGC; *: statistically significant.

Genetic alterations are frequently identified in EBV-associated tumors. Key genes associated with tumorigenesis often implicated, including the oncogenes PIK3CA, CCND1, LTBR and the tumor suppressor genes RASSF1A, NFKBIA, TRAF3, and TP53 (Tsao et al., 2017). Microtubule-associated protein 9 (MAP9, also known as ASAP) is a cell cycle-associated gene required for bipolar spindle assembly, mitosis progression and cytokinesis. Dysregulation of MAP9 expression leads to abnormal spindles with chromosome congression and segregation defects (Saffin et al., 2005; Venoux et al., 2008). New evidence demonstrates that MAP9 directly interacts with the $\mathrm{N}$-terminus of p53 in response to DNA damage. In doing so, it helps to stabilize p53 and promote p53-dependent transcription (Basbous et al., 2012). Thus, MAP9 is a newly discovered DNA damage receptor component, and its mutation or dysregulation is closely related to tumorigenesis. The MAP9 single nucleotide polymorphism (SNP) rs1058992 $(\mathrm{T}>\mathrm{C}$ ) is located in exon 11, and this missense mutation leads to the conversion of amino acids from lysine to arginine. Nevertheless, to date there have been no reports on the clinical significance of rs1058992.

In the present study, we analyzed SNP rs1058992 in EBV-positive/-negative NPC (EBV+/-NPC), EBVaGC,
EBV-negative GC (EBVnGC), and EBV-positive/negative lymphoma (EBV+/-L) samples. The homozygous $\mathrm{C}$ allele of the MAP9 SNP rs1058992 (T > C) was shown to be associated with increased risk of EBVaGC. This is the first report on the pathology of rs1058992.

\section{Materials and Methods}

Specimens. A total of 378 case patients and 100 populationbased control subjects were enrolled in our study, including 152 cases of GC (58 EBV positive and $94 \mathrm{EBV}$ negative), 98 cases of NPC (88 EBV positive and $10 \mathrm{EBV}$ negative) and 128 cases of lymphomas ( $86 \mathrm{EBV}$ positive and $42 \mathrm{EBV}$ negative). The paraffinembedded tissues were obtained from the Affiliated Hospital of Qingdao University and the Qingdao Municipal Hospital between 2008 and 2016. EBV-positive specimens were validated by in situ hybridization for EBV-encoded small RNA 1 (EBER1) and by PCR for EBV nuclear antigen 1 (EBNA1) in previous studies (Wang et al., 2010; Nie et al., 2013; Zhu et al., 2013; Sun et al., 2015). Peripheral blood samples from 100 healthy individuals with no history of gastric, nasopharyngeal, or lymphatic diseases were included as normal controls. This study was approved by 
Table 2. Genotype and allelic frequencies of the MAP9 polymorphism for nasopharyngeal cancer patients and normal controls

\begin{tabular}{|c|c|c|c|}
\hline MAP9/rs1058992 & $\begin{array}{c}\text { EBV negative NPC }(n=10) \\
n(\%)\end{array}$ & $\begin{array}{l}\mathrm{NC}(\mathrm{n}=100) \\
\mathrm{n}(\%)\end{array}$ & $\begin{array}{c}\text { EBV positive NPC }(\mathbf{n}=\mathbf{8 8}) \\
\mathbf{n}(\%)\end{array}$ \\
\hline \multicolumn{4}{|l|}{ genotype } \\
\hline $\mathrm{TT}$ & $1(10.00)$ & $14(14.00)$ & $11(12.50)$ \\
\hline CT & $4(40.00)$ & $45(45.00)$ & $38(43.18)$ \\
\hline OR (95\%CI) & $1.244(0.128-12.069)^{\mathrm{a}}$ & $0.864(0.087-8.543)^{c}$ & $1.075(0.437-2.644)^{\mathrm{b}}$ \\
\hline$P$ & $1.000^{\mathrm{a}}$ & $1.000^{c}$ & $0.875^{\mathrm{b}}$ \\
\hline $\mathrm{CC}$ & $5(50.00)$ & $41(41.00)$ & $39(44.32)$ \\
\hline OR $(95 \% \mathrm{CI})$ & $1.366(0.141-13.270)^{\mathrm{a}}$ & $0.709(0.075-6.720)^{c}$ & $1.211(0.491-2.987)^{\mathrm{b}}$ \\
\hline P & $1.000^{\mathrm{a}}$ & $1.000^{c}$ & $0.678^{\mathrm{b}}$ \\
\hline \multicolumn{4}{|l|}{ recessive model } \\
\hline $\mathrm{TT}+\mathrm{CT}$ & $5(50.00)$ & $59(59.00)$ & $49(55.68)$ \\
\hline CC & $5(50.00)$ & $41(41.00)$ & $39(44.32)$ \\
\hline OR $(95 \% \mathrm{CI})$ & $1.439(0.391-5.291)^{\mathrm{a}}$ & $0.796(0.215-2.947)^{c}$ & $1.145(0.642-2.044)^{\mathrm{b}}$ \\
\hline$P$ & $0.739^{\mathrm{a}}$ & $0.750^{c}$ & $0.646^{\mathrm{b}}$ \\
\hline \multicolumn{4}{|l|}{ dominant model } \\
\hline $\mathrm{TT}$ & $1(10.00)$ & $14(14.00)$ & $11(12.50)$ \\
\hline $\mathrm{CC}+\mathrm{CT}$ & $9(90.00)$ & $86(86.00)$ & $77(87.50)$ \\
\hline OR $(95 \% \mathrm{CI})$ & $1.465(0.172-12.475)^{\mathrm{a}}$ & $0.778(0.090-6.747)^{c}$ & $1.140(0.488-2.659)^{\mathrm{b}}$ \\
\hline$P$ & $1.000^{\mathrm{a}}$ & $1.000^{c}$ & $0.762^{\mathrm{b}}$ \\
\hline \multicolumn{4}{|l|}{ allele } \\
\hline $\mathrm{T}$ & $6(30.00)$ & $73(36.50)$ & $60(35.09)$ \\
\hline $\mathrm{C}$ & $14(70.00)$ & $127(63.50)$ & $116(65.91)$ \\
\hline OR $(95 \% \mathrm{CI})$ & $1.341(0.494-3.641)^{\mathrm{a}}$ & $0.829(0.303-2.266)^{c}$ & $1.111(0.727-1.699)^{\mathrm{b}}$ \\
\hline$P$ & $0.563^{\mathrm{a}}$ & $0.714^{c}$ & $0.626^{\mathrm{b}}$ \\
\hline
\end{tabular}

NPC: nas,opharyngeal carcinoma; NC: normal control; OR: odds ratio; 95\% CI: 95\% confidence interval; a: EBV-negative NPC vs. NC; b: EBV positive NPC vs. NC; c: EBV-positive NPC vs. EBV-negative NPC.

the Medical Ethical Committee of the Medical College, Qingdao University (QDU20160732). The tumor tissues and healthy peripheral blood samples were collected after written informed consent was obtained from all subjects.

DNA extraction. Genomic DNA was extracted from the paraffinembedded carcinoma tissues with a QIAamp DNA FFPE tissue kit (QIAGEN GmbH, Hilden, Germany). DNA was extracted from blood samples using the standard phenol-chloroform extraction procedure. DNA quality and quantity were measured with a NanoPhotometer P360 (Implen GmbH, Munich, Germany).

Genotyping. The concentration of the DNA samples was adjusted to 50 to $500 \mathrm{ng} / \mu \mathrm{l}$ prior to Sequenom MassARRAY analysis (Agena Biosciences, San Diego, CA, USA) to detect the rs 1058992 genotype. Matrix-assisted laser desorption ionization-time of flight mass spectrometry was used to analyze the products on the SpectroCHIPs produced by the MassARRAY. Sequenom TYPER 4.0 genotyping software was used to display the genotyping data. Allele frequencies were calculated from the genotype data.

Statistical analyses. The chi-squared test and correction for continuity were used to compare the differences in genotype and allele frequencies between each group. Non-conditional logistic regression was used to assess the odds ratio (OR). Correlations were considered statistically significant when $P<0.05$. Statistical analyses were performed with the SPSS 21.0 statistical software (IBM, New York, NY, USA).

\section{Results}

\section{Association of rs1058992 with gastric cancer}

A total of 58 cases of EBVaGC and 94 cases of EBVnGC were screened. The distribution of the three genotypes (TT, TC, and CC) and mutant allele frequencies for patients with gastric cancer and for the normal controls are shown in Table 1.

First, we compared the data between the EBVaGC and EBVnGC groups. The distribution of the three genotypes between EBVaGC and EBVnGC was significantly different $\left(\chi^{2}=7.687, P=0.021\right)$. Although not statistically significant, there were more CC genotype carriers $(P=0.091)$ in the EBVaGC group (56.90\%) compared with the EBVnGC group (34.04\%). Furthermore, carriers of the CC genotype of rs 1058992 had an increased risk of EBVaGC when compared with the reference $(\mathrm{TT}+\mathrm{CT})$ genotypes in the recessive model $(\mathrm{OR}=2.558,95 \% \mathrm{CI}=1.306-5.01, P=0.006)$. In the 
Table 3. Genotype and allelic frequencies of the MAP9 polymorphisms for lymphoma patients and normal controls

\begin{tabular}{|c|c|c|c|}
\hline MAP9/rs1058992 & $\begin{array}{c}\text { EBV-L }(n=42) \\
n(\%)\end{array}$ & $\begin{array}{c}\text { NC }(\mathbf{n}=100) \\
n(\%)\end{array}$ & $\begin{array}{c}\mathrm{EBV}+\mathrm{L}(\mathrm{n}=\mathbf{8 6}) \\
\mathrm{n}(\%)\end{array}$ \\
\hline \multicolumn{4}{|l|}{ genotype } \\
\hline TT & $5(11.90)$ & $14(14.00)$ & $13(15.12)$ \\
\hline $\mathrm{CT}$ & $16(38.10)$ & $45(45.00)$ & $39(45.35)$ \\
\hline OR $(95 \% \mathrm{CI})$ & $0,996(0.309-3.207)^{\mathrm{a}}$ & $0.938(0.287-3.064)^{c}$ & $0.933(0.392-2.224)^{b}$ \\
\hline$P$ & $1.000^{\mathrm{a}}$ & $0.915^{c}$ & $0.876^{\mathrm{b}}$ \\
\hline $\mathrm{CC}$ & $21(50.00)$ & $41(41.00)$ & $34(39.53)$ \\
\hline OR $(95 \% \mathrm{CI})$ & $1.434(0.455-4.523)^{\mathrm{a}}$ & $0.623(0.194-1.998)^{\mathrm{c}}$ & $0.893(0.370-2.156)^{\mathrm{b}}$ \\
\hline$P$ & $0.537^{\mathrm{a}}$ & $0.424^{c}$ & $0.801^{\mathrm{b}}$ \\
\hline \multicolumn{4}{|l|}{ recessive model } \\
\hline $\mathrm{TT}+\mathrm{CT}$ & $21(50.00)$ & $59(59.00)$ & $52(60.47)$ \\
\hline CC & $21(50.00)$ & $41(41.00)$ & $34(39.53)$ \\
\hline OR $(95 \% \mathrm{CI})$ & $1.439(0.697-2.969)^{\mathrm{a}}$ & $0.654(0.311-1.375)^{\mathrm{c}}$ & $0.941(0.523-1.694)^{\mathrm{b}}$ \\
\hline$P$ & $0.324^{\mathrm{a}}$ & $0.261^{\mathrm{c}}$ & $0.839^{\mathrm{b}}$ \\
\hline \multicolumn{4}{|l|}{ dominant model } \\
\hline $\mathrm{TT}$ & $5(11.90)$ & $14(14.00)$ & $13(15.12)$ \\
\hline $\mathrm{CC}+\mathrm{CT}$ & $37(88.10)$ & $86(86.00)$ & $73(84.88)$ \\
\hline OR (95\%CI) & $1.205(0.404-3.588)^{\mathrm{a}}$ & $0.759(0.251-2.290)^{\mathrm{c}}$ & $0.914(0.404-2.069)^{\mathrm{b}}$ \\
\hline$P$ & $0.738^{\mathrm{a}}$ & $0.624^{c}$ & $0.829^{\mathrm{b}}$ \\
\hline \multicolumn{4}{|l|}{ allele } \\
\hline $\mathrm{T}$ & $26(30.95)$ & $73(36.50)$ & $65(37.79)$ \\
\hline $\mathrm{C}$ & $58(69.05)$ & $127(63.50)$ & $107(62.21)$ \\
\hline OR (95\%CI) & $1.282(0.744-2.211)^{\mathrm{a}}$ & $0.738(0.423-1.287)^{c}$ & $0.946(0.621-1.443)^{b}$ \\
\hline$P$ & $0.371^{\mathrm{a}}$ & $0.283^{c}$ & $0.797^{\mathrm{b}}$ \\
\hline
\end{tabular}

NC: normal control; OR: odds ratio; 95\% CI: 95\% confidence interval; a: EBV-negative lymphoma vs. NC; b: EBV-positive lymphoma vs. NC; c: EBVpositive lymphoma vs. EBV-negative lymphoma.

dominant model, the increased risk of the CC + TC genotypes was not significant $(\mathrm{OR}=1.789,95 \% \mathrm{CI}=0.542-5.908$, $P=0.335)$. We also examined the correlation of $\mathrm{C}$ allele frequency with EBVaGC and EBVnGC. The presence of the $\mathrm{C}$ allele increased the risk of EBVaGC (OR $=1.904,95 \%$ $\mathrm{CI}=1.141-3.179, P=0.013)$.

When compared with the control group, higher homozygote CC frequency was observed in the EBVaGC group, but the difference was not significant $(P=0.083)$. Analysis of the dominant model and recessive model of the $\mathrm{C}$ allele also did not yield any significant differences. However, the $\mathrm{C}$ allele frequency was significantly increased $(\mathrm{OR}=1.724$, 95\% CI $=1.036-2.870, P=0.035)$. The genotype and allele frequency distributions of rs 1058992 did not differ between the EBVnGC and control groups.

SNP rs1058992 association with nasopharyngeal carcinoma and lymphoma

Additionally, we analyzed $88 \mathrm{EBV}$ positive NPC cases, 10 EBV negative NPC cases, $86 \mathrm{EBV}$ positive lymphoma cases, and $42 \mathrm{EBV}$ negative lymphoma cases. The associations of rs1058992 with NPC and lymphoma are shown in Tables 2 and 3, respectively. There were no differences observed in the genotype distribution and allele frequencies between any of these groups or between these groups and the control.

\section{Discussion}

In this study, we evaluated SNP rs1058992 in the MAP9 microtubule-associated gene, as this missense mutation may influence the function of its protein product and potentially contribute to carcinogenesis. The CC genotype was shown to be significantly associated with an increased risk of EBVaGC in the recessive model, and the $\mathrm{C}$ allele frequency was also significantly increased in EBVaGC group.

MAP9 is a member of the microtubule-associated protein family that regulates microtubule dynamics (Eot-Houllier et al., 2010). The MAPs act within the microtubule network regulating microtubule properties and functions, thereby participating in diverse cellular activities (Glotzer, 2009). MAP9 is phosphorylated by Aurora kinase A (AURKA) and polo-like kinase 1 (Plk1) during cell mitosis and is essential for spindle assembly, centrosome regulation, and cell division (Saffin et al., 2005; Venoux et al., 2008). The expression 
of MAP9 is downregulated in colorectal cancer, whereas AURKA and Plk1 are upregulated (Rouquier et al., 2014). Dysregulation of AURKA and Plk1 are strongly associated with tumorigenesis (Weng Ng et al., 2016; Al-Khafaji et al., 2017; Chen et al., 2017; Zhu et al., 2017), and AURKA polymorphisms have also been reported in association with GC and breast cancer (Lopez-Cortes et al., 2018; Mesic et al., 2017). Considering the direct interactions of MAP9 with AURKA and Plk1, we believe that MAP9 plays key role in carcinogenesis.

AURKA and Plk1 were also found to be differentially expressed in EBV-transformed lymphoblasts ( $\mathrm{Ng}$ et al., 2011; Dai et al., 2012;). The overexpression of AURKA and Plk1 may be driven by EBV LMP-1 (Ng et al., 2011). Studies have shown that in EBVaGC, EBNA2 disrupts mitotic checkpoints by upregulating Plk1 and downregulating mitotic arrest deficient-2 (MAD2) (Pan et al., 2009). However, the specific mechanisms of their deregulation in EBV-associated tumors remain unknown. MAP9, as a newly discovered Plk1 substrate that interacts with and stabilizes $\mathrm{p} 53$ protein, may be a key protein in the cell cycle of EBV-associated tumor cells. Nevertheless, until now there have been no reports regarding the role of MAP9 in EBV-associated tumor carcinogenesis.

For the first time, the SNP rs1058992 in the MAP9 gene was analyzed in three types of EBV-associated tumors. This SNP is located in exon 11 of the MAP9 gene and comprises a nucleotide substitution from $\mathrm{T}$ to $\mathrm{C}$, which changes the amino acid residue at this position from lysine to arginine. Our results demonstrated that the frequencies of the homozygous CC genotype and the $\mathrm{C}$ allele were significantly increased in the EBVaGC group compared with both the EBVnGC and control groups, suggesting that the CC genotype and $\mathrm{C}$ allele are risk factors for EBVaGC. The results of an ongoing project in our laboratory have shown that MAP9 is silenced in gastric cancer cells as a result of promoter methylation; however, MAP9 is expressed in EBV-positive GC cells (unpublished data). Although it is too early to draw any conclusions, this missense mutation may lead to structural and functional changes in the MAP9 protein.

Interestingly, $\mathrm{C}$ allele carriers did not show increased susceptibility towards EBV-positive NPC even though NPC is also an epithelial tumor. However, this result may be due to the limited number of EBV-negative samples in our study. Because EBV is extensively found in cases of NPC, only 10 cases of EBV-negative NPC were available for this study. We also characterized rs1058992 in EBV-positive/EBV-negative lymphoma. No significant differences in genotype and allele frequency were observed for lymphoma either; this may be due to the distinct expression pattern of EBV latent genes in GC.

In conclusion, the present study revealed that the homozygous (CC) genotype of the MAP9 rs1058992 polymorphism is significantly associated with increased risk of EBVaGC, as is the frequency of the $\mathrm{C}$ allele. These observations indicate the possible role of the MAP9 gene in mitotic checkpoints and EBV-associated tumorigenesis.

Acknowledgments. This research was supported by the Natural Science Foundation of Shandong Province (ZR2017BH106) and the China Postdoctoral Science Foundation (2017M610411).

\section{References}

Al-Khafaji ASK, Marcus MW, Davies MPA, Risk JM, Shaw RJ, Field JK, Liloglou T (2017): AURKA mRNA expression is an independent predictor of poor prognosis in patients with non-small cell lung cancer. Oncol. Lett. 13, 4463-4468. https://doi.org/10.3892/ol.2017.6012

Ali AS, Al-Shraim M, Al-Hakami AM, Jones IM (2015): EpsteinBarr Virus: Clinical and Epidemiological Revisits and Genetic Basis of Oncogenesis. Open Virol. J 9, 7-28. https://doi.org/10.2174/1874357901509010007

Basbous J, Knani D, Bonneaud N, Giorgi D, Brondello JM, Rouquier S (2012): Induction of ASAP (MAP9) contributes to 553 stabilization in response to DNA damage. Cell Cycle 11, 2380-2390. https://doi.org/10.4161/cc.20858

Camargo MC, Kim WH, Chiaravalli AM, Kim KM, Corvalan AH, Matsuo K, Yu J, Sung JJ, Herrera-Goepfert R, MenesesGonzalez F (2014): Improved survival of gastric cancer with tumour Epstein-Barr virus positivity: an international pooled analysis. Gut 63, 236-243. https://doi. org/10.1136/gutjnl-2013-304531

Cancer Genome Atlas Research N (2014): Comprehensive molecular characterization of gastric adenocarcinoma. Nature 513, 202-209. https://doi.org/10.1038/nature13480

Chen C, Song G, Xiang J, Zhang H, Zhao S, Zhan Y (2017): AURKA promotes cancer metastasis by regulating epithelialmesenchymal transition and cancer stem cell properties in hepatocellular carcinoma. Biochem. Biophys. Res. Commun. 486, 514-520. https://doi.org/10.1016/j. bbrc.2017.03.075

Dai Y, Tang Y, He F, Zhang Y, Cheng A, Gan R, Wu Y (2012): Screening and functional analysis of differentially expressed genes in EBV-transformed lymphoblasts. Virol. J. 9, 77. https://doi.org/10.1186/1743-422X-9-77

Elgui de Oliveira D, Muller-Coan BG, Pagano JS (2016): Viral Carcinogenesis Beyond Malignant Transformation: EBV in the Progression of Human Cancers. Trends Microbiol. 24, 649-664. https://doi.org/10.1016/j.tim.2016.03.008

Eot-Houllier G, Venoux M, Vidal-Eychenie S, Hoang MT, Giorgi D, Rouquier S (2010): Plk1 regulates both ASAP localization and its role in spindle pole integrity. J. Biol. Chem. 285, 29556-29568. https://doi.org/10.1074/jbc.M110.144220

Ferlay J, Soerjomataram I, Dikshit R, Eser S, Mathers C, Rebelo M, Parkin DM, Forman D, Bray F (2015): Cancer incidence and mortality worldwide: sources, methods and major patterns in GLOBOCAN 2012. Int. J. Cancer 136, E359-386. https://doi.org/10.1002/ijc.29210 
Glotzer M (2009): The 3Ms of central spindle assembly: microtubules, motors and MAPs. Nat. Rev. Mol. Cell Biol. 10, 9-20. https://doi.org/10.1038/nrm2609

Huang SC, Ng KF, Chen KH, Hsu JT, Liu KH, Yeh TS, Chen TC (2014): Prognostic factors in Epstein-Barr virus-associated stage I-III gastric carcinoma: implications for a unique type of carcinogenesis. Oncol. Rep. 32, 530-538. https:// doi.org/10.3892/or.2014.3234

Ko YH (2015): EBV and human cancer. Exp. Mol. Med. 47, e130. https://doi.org/10.1038/emm.2014.109

Lopez-Cortes A, Cabrera-Andrade A, Ona-Cisneros F, Rosales F, Ortiz M, Tejera E, Paz YMC (2018): Breast Cancer Risk Associated with Genotype Polymorphisms of the Aurora Kinase a Gene (AURKA): a Case-Control Study in a High Altitude Ecuadorian Mestizo Population. Pathol. Oncol. Res. 24, 457-465 https://doi.org/10.1007/ s12253-017-0267-6

Mesic A, Markocic E, Rogar M, Juvan R, Hudler P, Komel R (2017): Single nucleotide polymorphisms rs911160 in AURKA and rs2289590 in AURKB mitotic checkpoint genes contribute to gastric cancer susceptibility. Environ. Mol. Mutagen. 58, 701-711. https://doi.org/10.1002/em.22129

Ng SB, Selvarajan V, Huang G, Zhou J, Feldman AL, Law M, Kwong YL, Shimizu N, Kagami Y, Aozasa K (2011): Activated oncogenic pathways and therapeutic targets in extranodal nasal-type NK/T cell lymphoma revealed by gene expression profiling. J. Pathol. 223, 496-510. https://doi. org/10.1002/path.2823

Nie Y, Sun Y, Wang Y, Liu C, Zhao C, Luo B (2013): Epstein-Barr virus gene polymorphism in different parts of the same nasopharyngeal carcinoma patient. Arch. Virol. 158, 1031-1037. https://doi.org/10.1007/s00705-012-1578-2

Pan SH, Tai CC, Lin CS, Hsu WB, Chou SF, Lai CC, Chen JY, Tien HF, Lee FY, Wang WB (2009): Epstein-Barr virus nuclear antigen 2 disrupts mitotic checkpoint and causes chromosomal instability. Carcinogenesis 30, 366-375. https:// doi.org/10.1093/carcin/bgn291

Rosales-Perez S, Cano-Valdez AM, Flores-Balcazar CH, GuedeaEdo F, Lino-Silva L S, Lozano-Borbalas A, NavarroMartin A, Poitevin-Chacon A (2014): Expression of Epstein-Barr virus-encoded latent membrane protein (LMP-1), p16 and p53 proteins in nonendemic nasopharyngeal carcinoma (NPC): a clinicopathological study. Arch. Med. Res. 45, 229-236. https://doi.org/10.1016/j. arcmed.2014.02.002

Rouquier S, Pillaire MJ, Cazaux C, Giorgi D (2014): Expression of the microtubule-associated protein MAP9/ASAP and its partners AURKA and PLK1 in colorectal and breast cancers. Dis. Markers 2014, 798170. https://doi. org/10.1155/2014/798170
Saffin JM, Venoux M, Prigent C, Espeut J, Poulat F, Giorgi D, Abrieu A, Rouquier S (2005): ASAP, a human microtubule-associated protein required for bipolar spindle assembly and cytokinesis. Proc. Natl. Acad. Sci. USA 102, 11302-11307. https://doi.org/10.1073/pnas.0500964102

Sun L, Zhao Z, Liu S, Liu X, Sun Z, Luo B (2015): Sequence Variation Analysis of Epstein-Barr Virus Nuclear Antigen 1 Gene in the Virus Associated Lymphomas of Northern China. PLoS One 10, e0140529. https://doi.org/10.1371/ journal.pone.0140529

Tanyildiz HG, Yildiz I, Bassullu N, Tuzuner N, Ozkan A, Celkan T, Apak H (2015): The Role of Epstein-Barr Virus LMP-1 Immunohistochemical Staining in Childhood Hodgkin Lymphoma. Iran. J. Pediatr. 25, e2359. https://doi. org/10.5812/ijp.2359

Tsao SW, Tsang CM, Lo KW (2017): Epstein-Barr virus infection and nasopharyngeal carcinoma. Philos. Trans. R. Soc. Lond. B. Biol. Sci. 372. https://doi.org/10.1098/ rstb.2016.0270

Venoux M, Basbous J, Berthenet C, Prigent C, Fernandez A, Lamb NJ, Rouquier S (2008): ASAP is a novel substrate of the oncogenic mitotic kinase Aurora-A: phosphorylation on Ser625 is essential to spindle formation and mitosis. Hum. Mol. Genet. 17, 215-224. https://doi.org/10.1093/ $\underline{\mathrm{hmg} / \mathrm{ddm} 298}$

Wang Y, Liu X, Xing X, Cui Y, Zhao C, Luo B (2010): Variations of Epstein-Barr virus nuclear antigen 1 gene in gastric carcinomas and nasopharyngeal carcinomas from Northern China. Virus Res. 147, 258-264. https://doi.org/10.1016/j. virusres.2009.11.010

Weng Ng WT, Shin JS, Roberts TL, Wang B, Lee CS (2016): Molecular interactions of polo-like kinase 1 in human cancers. J. Clin. Pathol. 69, 557-562. https://doi.org/10.1136/ jclinpath-2016-203656

Wu R, Sattarzadeh A, Rutgers B, Diepstra A, van den Berg A, Visser L (2016): The microenvironment of classical Hodgkin lymphoma: heterogeneity by Epstein-Barr virus presence and location within the tumor. Blood Cancer J. 6, e417. https://doi.org/10.1038/bcj.2016.26

Young LS, Rickinson AB (2004): Epstein-Barr virus: 40 years on. Nat. Rev. Cancer 4, 757-768. https://doi.org/10.1038/nrc1452

Zhu L, Xing S, Zhang L, Yu JM, Lin C, Yang WJ (2017): Involvement of Polo-like kinase 1 (Plk1) in quiescence regulation of cancer stem-like cells of the gastric cancer cell lines. Oncotarget 8, 37633-37645. https://doi.org/10.18632/ oncotarget.16839

Zhu S, Sun P, Zhang Y, Yan L, Luo B (2013): Expression of c-myc and PCNA in Epstein-Barr virus-associated gastric carcinoma. Exp. Ther. Med. 5, 1030-1034. https://doi. org/10.3892/etm.2013.972 